\title{
Safety evaluation of high-risk myocardial micro-biopsy in a swine model
}

\author{
Arvin Chireh $^{1} \oplus \cdot$ Mikael Sandell $^{1,2,3} \cdot$ Rikard Grankvist $^{1} \cdot$ Victoria Lövljung ${ }^{1} \cdot$ Jonathan al-Saadi $^{1} \cdot$ Fabian Arnberg $^{1,4}$. \\ Johan Lundberg ${ }^{1,4} \cdot$ Magnus Settergren $^{5,6} \cdot$ Staffan Holmin ${ }^{1,4}$
}

Received: 28 July 2021 / Accepted: 12 November 2021 / Published online: 23 November 2021

(c) The Author(s) 2021

\begin{abstract}
The objective of the study was to investigate the safety profile of high-risk micro-endomyocardial biopsy (micro-EMB) compared to conventional EMB in a large animal model. Twenty pigs were subjected to a maximum of 30 consecutive biopsies, including sampling from the free ventricular wall, with either micro-EMB $(n=10)$ or conventional EMB $(n=10)$. There were no major complications in the micro-EMB group (0/10), compared to six major complications in the EMB group (6/10; $p=0.003)$. Survival analysis further highlighted these differences $(p=0.004)$. There were significantly higher volumes of pericardial effusion in the EMB group $(p=0.01)$. The study shows a safety advantage of micro-EMB compared to standard EMB in the experimental high-risk circumstances investigated in this animal study. These results indicate enhanced possibilities to collect samples from sensitive areas by using the micro-EMB technique instead of standard EMB.
\end{abstract}

Keywords Endomyocardial biopsy $\cdot$ Micro-biopsy $\cdot$ Safety $\cdot$ Interventional cardiology

$\begin{array}{ll}\text { Abbreviations } \\ \text { EMB } & \text { Endomyocardial biopsy } \\ \text { F } & \text { French } \\ \text { TTE } & \text { Transthoracic echocardiography } \\ \text { LV } & \text { Left ventricle } \\ \text { MAP } & \text { Mean artery pressure } \\ \text { PA } & \text { Posteroanterior } \\ \text { Px } & \text { Pericardial effusion } \\ \text { RV } & \text { Right ventricle }\end{array}$

Staffan Holmin

staffan.holmin@ki.se

1 Department of Clinical Neuroscience, Karolinska Institutet, Stockholm, Sweden

2 Division of Micro and Nanosystems, Royal Institute of Technology, Stockholm, Sweden

3 MedTechLabs, Solna, Sweden

4 Department of Neuroradiology, Karolinska University Hospital, Stockholm, Sweden

5 Department of Cardiology, Karolinska University Hospital, Stockholm, Sweden

6 Department of Medicine, Karolinska Institutet, Stockholm, Sweden

\section{Introduction}

Endomyocardial biopsy (EMB) is an established method to obtain cardiac biopsies through the endovascular route. The method is primarily used to detect allograft rejection (after cardiac transplantation) and for investigating selected cases of unexplained heart failure [1,2]. Despite the complication risks and a relatively low sensitivity, EMB is considered the reference method for diagnosis of several cardiac diseases, such as myocarditis $[2,3]$. Studies report a variable complication rate between less than $1 \%$ and $8.9 \%$, with cardiac tamponade and arrhythmias among the severe complications [4-7]. Despite agreement that bi-ventricular biopsies could improve sensitivity, left ventricle EMB (LV-EMB) is not widely considered safe, although recent data suggest relatively low risks when performed by experienced operators $[6,8,9]$.

To improve the safety and value of EMB, we previously developed a novel EMB device and analysis protocol, referred to as micro-EMB [10]. The novel method features a significantly miniaturized and flexible biopsy device coupled to low-input analysis methods such as RNA-sequencing. Our previous studies of LV-micro-EMB in swine showed favorable safety outcomes as well as other outcomes such as navigability, sample quality, and ability to detect disease related changes $[10,11]$. For instance, it was shown that each 
micro-EMB attempt caused an injured area of $0.3 \mathrm{~mm}^{2}$ in the endomyocardium, compared to $18 \mathrm{~mm}^{2}$ with a conventional device [10]. Although this minimized trauma suggests lower procedural risks, a dedicated safety study was not performed. Therefore, this study was conducted to investigate the safety profile of micro-EMB compared to standard EMB in a large animal model.

We hypothesized that micro-EMB will cause fewer major complications compared to conventional EMB. However, since severe complications from EMB are relatively rare (although clinically significant), an impractical number of animals would be required to detect potential differences between micro-EMB and conventional EMB with standard clinical protocols. Therefore, we further hypothesized that an aggressive sampling approach, including sampling from the free wall, would increase the number of events sufficiently to enable statistical analysis despite a relatively small number of animals.

To this end, we designed this study to compare the safety profiles of micro-EMB and standard EMB in a limited number of animals, using a high-risk sampling approach.

\section{Materials and methods}

\section{Experimental design}

Twenty mixed breed Yorkshire-Swedish farm pigs (Table 1, Online Resource 1) were subjected to either micro-EMB $(n=10)$ or EMB ( $n=10$; Fig. 1$)$, using a high-risk sampling approach. The animals were continuously monitored in the operating room, and transthoracic echocardiography (TTE) was performed after each biopsy attempt. A maximum of 30 biopsies were taken from various parts of the right ventricle, including the free wall. The experiment was terminated either upon a major complication or a maximum of 30 biopsies without any major complication. A major complication was defined as a cardiac event (such as arrhythmia or pericardial effusion) causing death or sustained hemodynamic compromise (mean artery pressure (MAP) below 45 for at least $20 \mathrm{~min}$ ). Hemodynamic compromise due to pericardial effusion was classified as tamponade. The frequencies and character of the severe complications were compared on group level between the two groups.

Table 1 Animal characteristics and outcome

\begin{tabular}{llll}
\hline Group & $\begin{array}{l}\text { Gender }(n \\
\text { female/total })\end{array}$ & $\begin{array}{l}\text { Weight (median } \\
\text { and range, } \mathrm{kg})\end{array}$ & $\begin{array}{l}\text { Outcome } \\
(n \text { events/ } \\
\text { total })\end{array}$ \\
\hline Micro-EMB & $7 / 10$ & $39.9(36.1-45.0)$ & $0 / 10$ \\
Conventional EMB & $7 / 10$ & $40.4(32.5-48.3)$ & $6 / 10$ \\
\hline
\end{tabular}

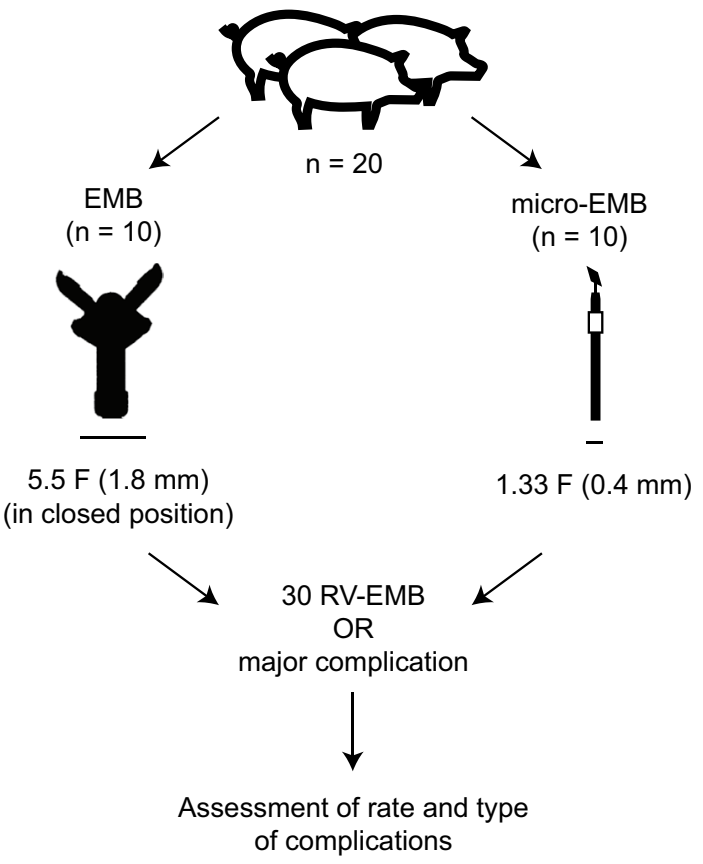

Fig. 1 Study design. Twenty pigs were subjected to either microEMB with a $0.4 \mathrm{~mm}$ micro-EMB device $(n=10)$ or EMB with a standard 5.5 F Cordis Biopsy Forceps $(n=10)$. A high-risk sampling schedule was employed, including a maximum of 30 biopsies taken from various parts of the right ventricle, including the free wall. The experiment was terminated either upon a major complication or 30 biopsies without any major complication. Acute complications were assessed and compared on group level

\section{Ethical considerations}

The study was conducted in accordance with national and local guidelines for Sweden and Karolinska Institutet, respectively. All animal experiments were approved from the local ethics committee (Stockholms Norra Djurförsöksetiska Nämnd, Stockholm, Sweden) prior to starting the experiments. All experiments were performed in accordance with 'Principles of Laboratory Animal Care' formulated by the National Society for Medical Research as well as the 'Guide for the Care and Use of Laboratory Animals' prepared by the Institute of Laboratory Animal Resources.

\section{Animal experiments}

The animals were allocated to each study group in an alternating order, so that approximately every other animal was allocated to the micro-EMB group and the other to the standard EMB group. There was no blinding of the operator or the rest of the staff. There were no predetermined inclusion or exclusion criteria.

All animals were anesthetized and monitored using the same protocol as previously reported [10]. Throughout the 
intervention, the animals were monitored for complications using continuous ECG, invasive blood pressure measurement, oxygen saturation, temperature, and urine production. Animals were treated with $75 \mathrm{mg}$ amiodarone intravenously (iv) as a single dose prior to cardiac catheterization and 5000 units of heparin per hour during the procedure.

Vascular access was gained by surgical cut-down of the left or right external jugular vein followed by catheterization (using Seldinger technique). An 8.5 French (F) Agilis $^{\mathrm{TM}}$ NxT steerable introducer (Abbott Vascular, Menlo Park, California, USA) was inserted and advanced to the $\mathrm{RV}$. The location was confirmed with ventriculography and pressure measurements through the introducer sheath. Micro-EMB samples were harvested from the myocardium using a similar technique as previously described, with two minor modifications [10]. First, the tip of the device was reduced to a total penetration depth of $2 \mathrm{~mm}$ (due to the reduced wall thickness of RV compared to LV). Second, the device was housed inside a guiding catheter (5F Envoy ${ }^{\circledR}$ with multi-purpose D tip, Codman Neuro, Raynham, Massachusetts, United States) instead of a microcatheter since there was already an introducer sheath placed in the RV. Conventional EMB samples were taken with a 5.5 F Cordis Biopsy Forceps (Cordis, Hialeah, Florida, United States), using standard clinical technique supervised by a consultant in interventional cardiology. All biopsies were initially directed towards the right interventricular septum followed by incremental variations to the sampling location. The bioptome was aimed towards the inferior, middle, and superior portions of the septum, as well as the anterior, lateral, and posterior free wall.

After each micro-EMB attempt, the device was assessed under light microscopy to ensure that a biopsy sample was taken. Cases with none or insufficient sample material were considered as failed biopsy attempts and were not counted as performed biopsies. After each biopsy (regardless of method), a quick echocardiography examination was performed to detect pericardial effusion (Px). A Philips ultrasound machine (CX 50) with an S5-1 cardiac probe was used, placing the probe in a left midaxillary position, aiming for a view resembling a human short-axis view.

When the endpoint was reached (major complication or 30 biopsies), the animal was euthanized with a lethal dose of pentobarbital. Sternotomy was performed to evaluate pericardial fluid and the heart was extracted for gross inspection.

\section{Data analysis and statistics}

All animals $(n=20)$ were included in the analysis. Descriptive statistics were used to assess distribution of weight and gender between the groups. Values are reported in median and range unless otherwise specified.
Chi-square test was used to test the overall difference between the groups in terms of complication frequencies. Kaplan-Meier survival analysis was performed using the "survival" package (version 3.2.3) in R. Major complications were classified as events. Animals were censored after 30 successful biopsy attempts. Log-rank test was used to determine statistical difference between the two groups in the survival analysis. Unpaired $t$ test was used to test the difference of pericardial effusion between the groups.

All statistical analyses were performed using the $\mathrm{R}$ statistical computing language, version 4.0.2. $p$ values of 0.05 or less were considered significant.

\section{Results}

Twenty swine (Table 1 ) were subjected to extensive biopsy sampling with either micro-EMB $(n=10)$ or $\operatorname{EMB}(n=10)$, including sampling from the free ventricular wall. In the micro-EMB group, 300 of 325 (92\%) biopsy attempts were successful, compared to 185 of 189 (98\%) in the EMB group. Failed biopsy attempts were not counted as performed biopsies. The number of major complications was $0 / 10(0 \%)$ in the micro-EMB group and 6/10 (60\%) in the conventional EMB group, which was significantly different between the groups $(p=0.003)$. All major complications were in the form of acute cardiac tamponade characterized by hypotension and pericardial effusion (Fig. 2).

Survival analysis was performed to investigate time-toevent (Fig. 3). Major complications, such as severe arrhythmia and tamponade, were considered as events, although only tamponade occurred. Animals were censored after 30 biopsies. The results showed significant survival differences between the groups ( $p=0.004, \log$-rank test). There were no events in the micro-EMB group, while the conventional group suffered from evenly distributed events.

After euthanasia, the volume and appearance of the pericardial fluid was assessed (Fig. 4). Blood-colored pericardial effusion was detected in 12 animals, of which seven were from the EMB group (58\%). Half of these animals $(n=6)$ had a known tamponade, with pericardial effusion ranging between 125 and $200 \mathrm{ml}$, whereas the others $(n=6)$ had an undetected perforation with a blood-colored effusion ranging between 10 and $43 \mathrm{ml}$. Overall, the volume of pericardial fluid was significantly higher in the conventional EMB group compared to the micro-EMB group $(p=0.01)$.

To further investigate the timing and location of the cardiac wall perforations, post-mortem tissues and transthoracic echocardiogram (TTE) examinations were reviewed. Post-mortem analysis of animals with pericardial blood $(n=12)$ revealed hematomas and suspected perforations on the anterior $(n=2)$, posterior $(n=7)$, or multiple $(n=3)$ aspects of the myocardial wall (Online Resource 1). On 
Fig. 2 Tamponade in an animal subjected to high-risk conventional EMB. a Transthoracic echocardiogram showed extensive pericardial effusion after 11 biopsies, while the animal was hypotensive. b Sternotomy after euthanasia revealed a bloodfilled pericardial sac, measuring $180 \mathrm{ml}$. c Closer inspection of the heart showed hematomas on the posterior surface of the right ventricle (RV), with a perforation (asterisk)
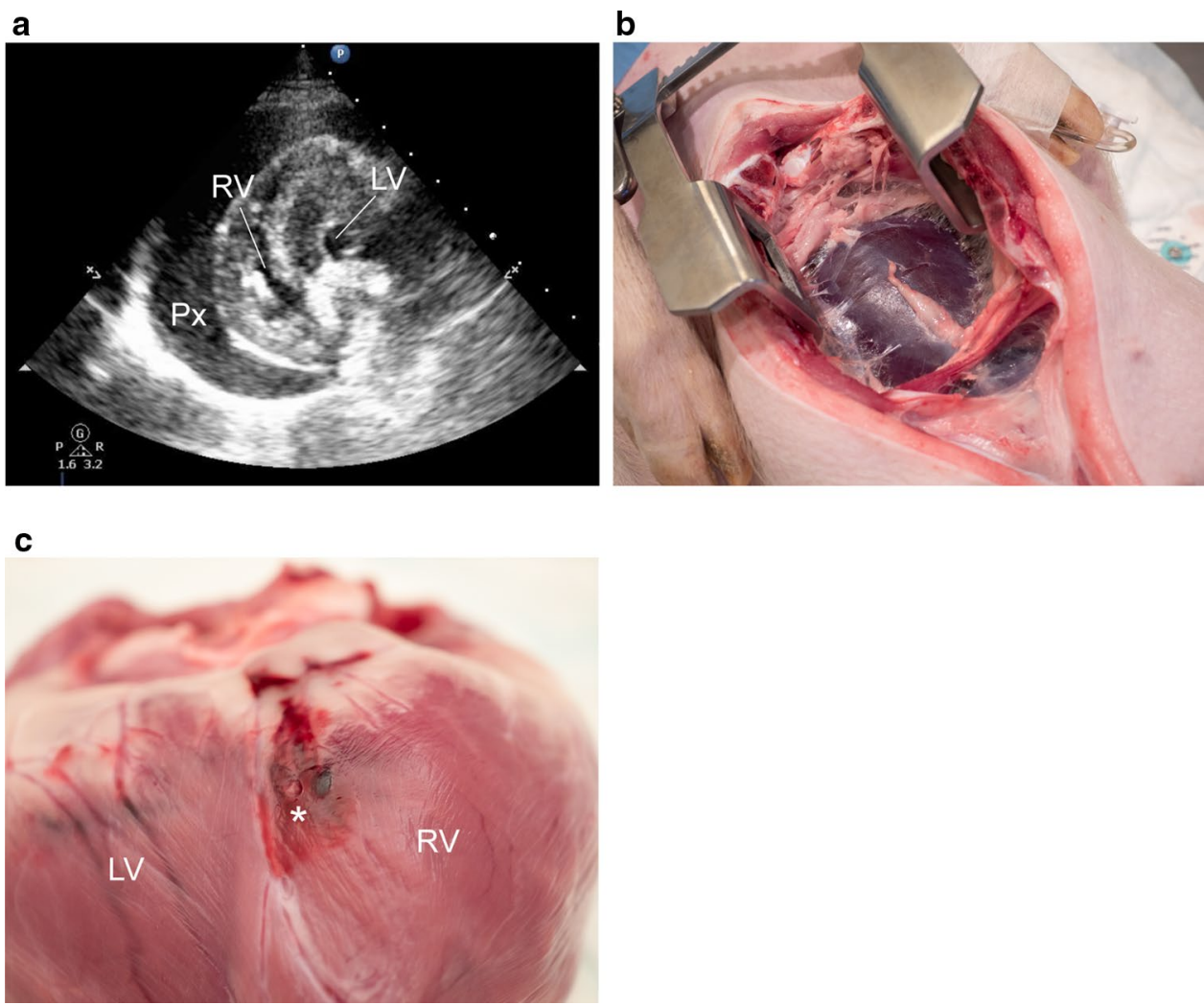

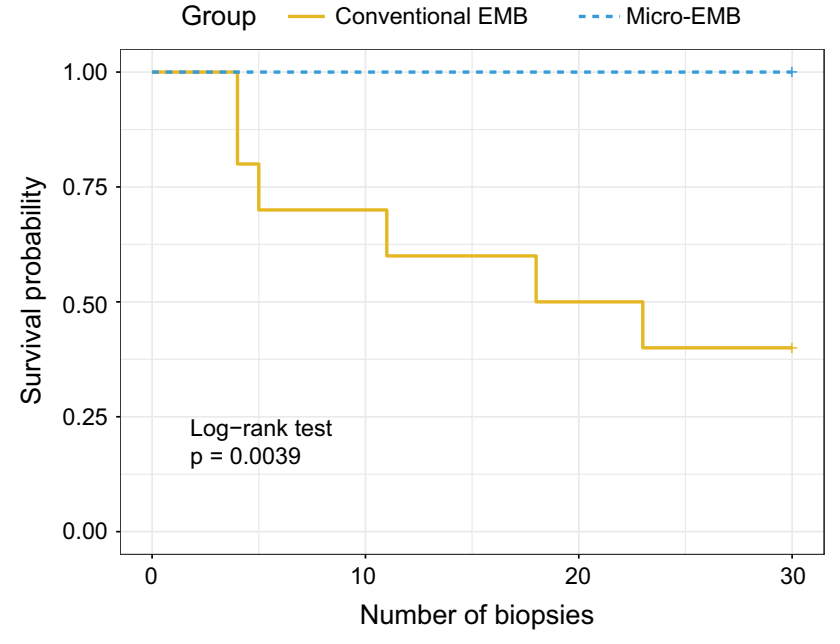

Fig. 3 Kaplan-Meier survival analysis. Survival plot shows a significant difference between micro-EMB and conventional EMB $(p=0.004)$ after high-risk biopsy sampling in swine $(n=20)$. Major complications such as severe arrhythmia or acute tamponade were considered as events. Animals were censored after 30 biopsies

TTE, pericardial effusion was detected in all animals with tamponade but not in the non-tamponade animals, impeding the possibility to determine the time (number of biopsies) to cardiac wall perforation in those cases. Examples of a

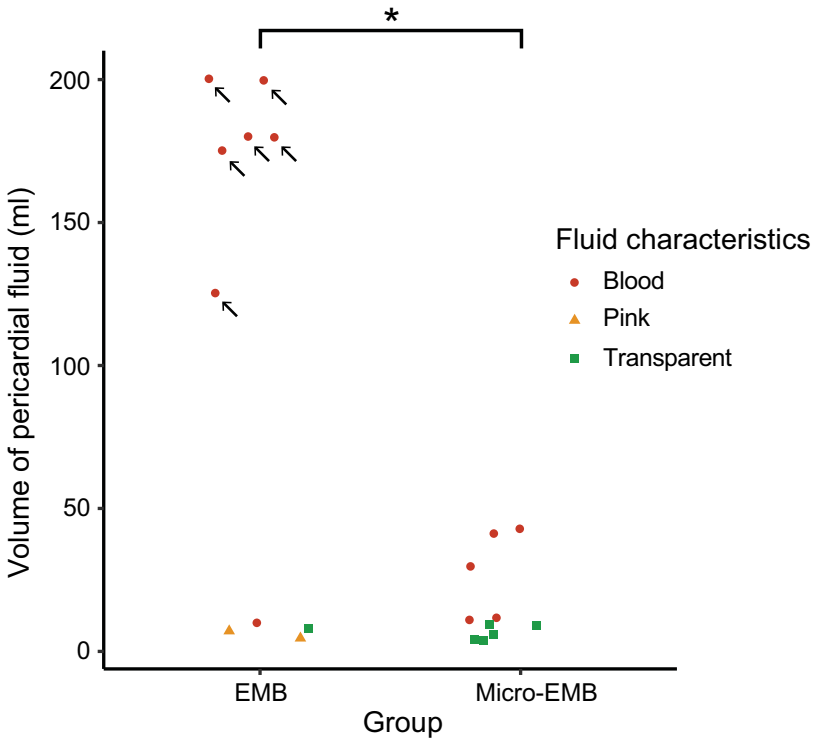

Fig. 4 Volume and characteristics of pericardial fluid post-mortem. Following the series of high-risk biopsy sampling ( $n=20$ animals), there were higher volumes of pericardial effusion (blood) in the EMB group compared to micro-EMB $(p=0.01)$. Animals indicated with arrows $(n=6)$ suffered from tamponade whereas the others were hemodynamically stable 
negative TTE examination and post-mortem hematoma in one animal subjected to micro-EMB are shown (Fig. 5).

To assess rates of arrhythmia, the animals were continuously monitored with ECG. All animals (in both groups) exhibited temporary tachycardia when penetrating the myocardium with the biopsy devices, but no animal suffered from prolonged arrhythmia with hemodynamic compromise.

As part of the study design, sampling location was varied after every five biopsies. Incidentally, it was noted that the conventional EMB device was difficult to accurately navigate due to stiffness and size of the bioptome (Fig. 6a, b). In comparison, micro-EMB was easier to direct and also featured a guide catheter with a bent tip to allow another dimension of movement (Fig. 6c, d).

\section{Discussion}

Micro-EMB was recently developed as a potential alternative to conventional EMB, aiming to increase flexibility and safety. In this study, we compared the safety profiles of micro-EMB and conventional EMB in a limited number of swine, using an experimental high-risk sampling approach. As hypothesized, there was a significantly lower number of major complications in the micro-EMB group compared to conventional EMB in these circumstances, despite the few animals enrolled. In the micro-EMB group, there was no major complication, while there was a tamponade in six of ten animals in the conventional EMB group. These results clearly suggest a safety advantage of micro-EMB
Fig. 5 Echocardiography and post-mortem analysis in one animal subjected to high-risk micro-EMB (30 biopsies). This animal was hemodynamically stable and had no detectable px on TTE (a), but post-mortem analysis revealed $11 \mathrm{ml}$ of blood-colored pericardial effusion. b Anterior wall of the RV shows hematoma. c Close-up of dashed area in (b) reveals two suspected perforations (asterisks). $\mathbf{d}$ The posterior wall of the RV also shows a hematoma with one suspected perforation (e, asterisk)

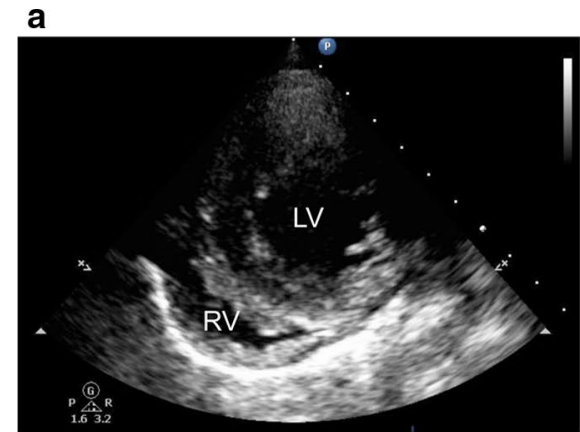

b

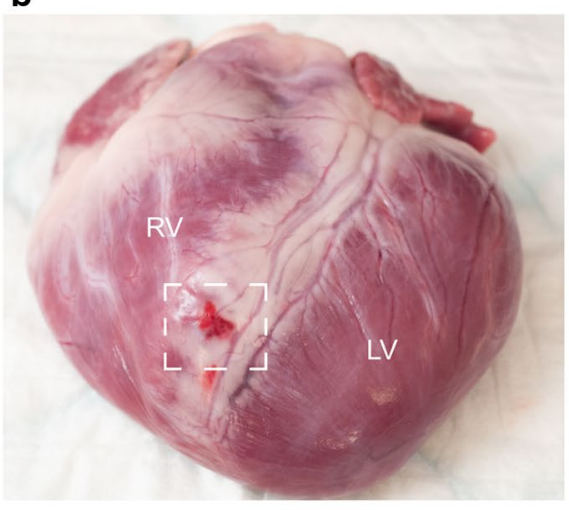

c

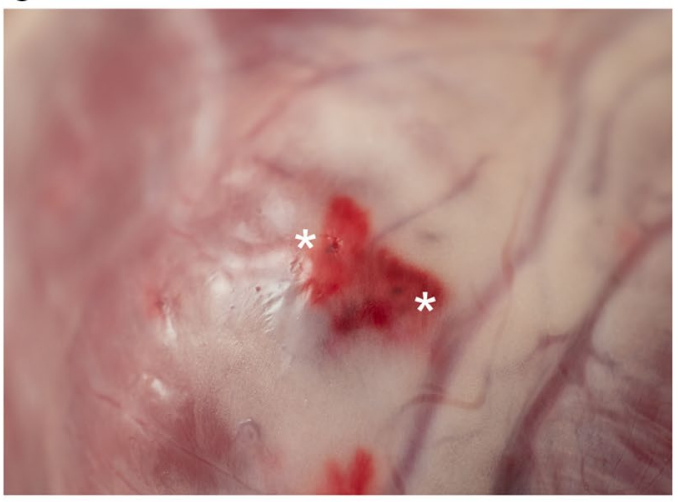

d

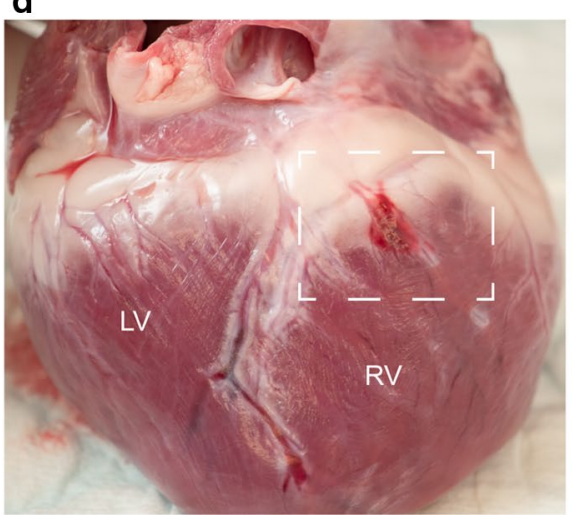

e

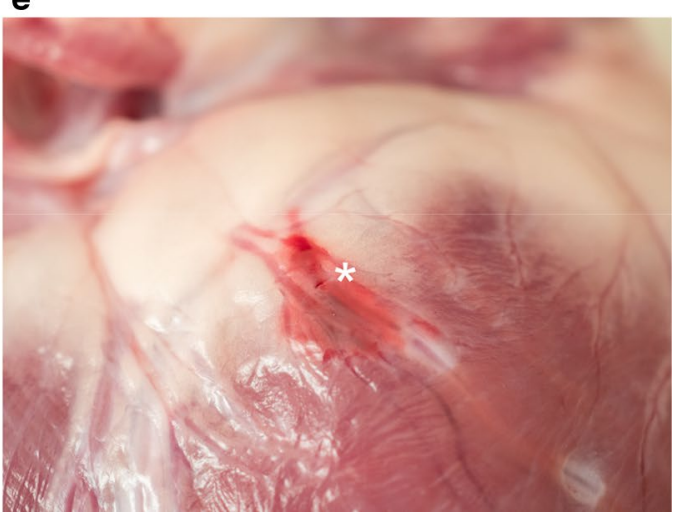


Fig. 6 Fluoroscopy images in PA projection, showing navigability differences between EMB and micro-EMB. a, b Shows how the flexible introducer sheath $(8.5 \mathrm{~F})$ changes angle after the conventional EMB bioptome is advanced (b). c, d Shows intact curvature of the introducer after advancing the $5 \mathrm{~F}$ guide catheter housing the micro-EMB device (d). The same introducer sheath was used $(8.5 \mathrm{~F})$, although with a slightly different curve. In $\mathbf{c}$ and d, the introducer is filled with contrast medium due to preceding ventriculography
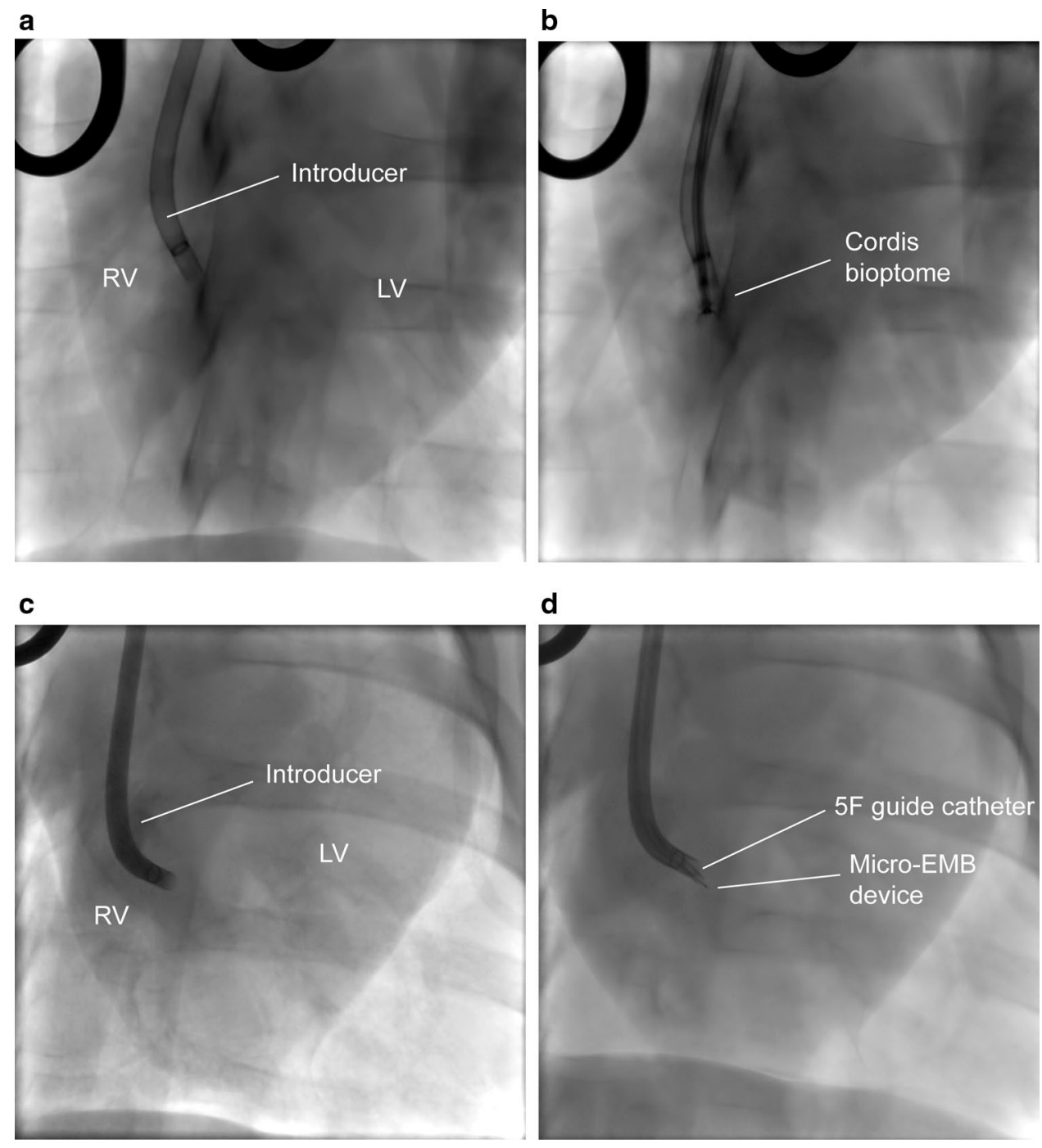

when performing extensive sampling from areas of the right ventricle that are prone to complications.

The high complication rate in the EMB group might appear surprising compared to available clinical data, which suggest a major complication rate of less than $1 \%$ [12]. However, as mentioned in the introduction, the highrisk sampling approach employed in this study was different from a standard clinical EMB procedure. We performed biopsies repeatedly from several regions of the myocardial wall, including the right ventricular free wall, which was expected to yield a higher complication frequency. This approach was selected to highlight potential differences between the methods without the need of a large number of animals. Despite the discrepancy between clinical protocols and the high-risk approach used in this study, the comparison is still relevant for a number of reasons. For instance, there are diseases with patchy distributions, or tumors, that cannot be detected by exclusively targeting the septum and can therefore require sampling from sensitive areas $[8,13,14]$. Moreover, repeated surveillance biopsies after cardiac transplantation can cause extensive fibrosis, making it increasingly difficult to obtain adequate samples without varying the sampling location $[15,16]$. Overall, since inadequate sampling is one of the main limitations of EMB [14, 17], the suggested safety advantage of micro-EMB in high-risk scenarios has the potential to widen the diagnostic utility compared to standard EMB.

Post-mortem analysis revealed pericardial effusion (blood) and macroscopic signs of perforation in animals from both groups. However, hemodynamic compromise did not occur in the micro-EMB group. Since these smaller effusions were only revealed post-mortem, the timing of the perforation could not be established. Given the high number of biopsies from varied locations, perforation at some point should not come as a surprise. This finding highlights that caution and appropriate monitoring is warranted regardless of device, but that the risk of perforation causing hemodynamic compromise was significantly 
higher with the conventional method in the studied circumstances.

Although arrhythmia is one of the feared complications of EMB, no severe arrhythmia with hemodynamic compromise occurred during the study. These results should be interpreted with caution since the animals were given antiarrhythmic drugs, due to known myocardial sensitivity in pig, as well as lack of follow-up.

Extensive handling of both micro-EMB and EMB devices revealed a flexibility advantage of micro-EMB, enabling more accurate sampling. The conventional EMB device is relatively difficult to navigate accurately due to size and stiffness, even when using a flexible introducer sheath. Although crude positional changes were still possible, the steering difficulties could be relevant in scenarios where an accurate targeting is needed, such as in patchy disease or extensive fibrosis. As discussed in our previous study, micro-EMB could potentially be used in conjunction with interventional MRI guidance, or other navigational tools, to refine accuracy even further [11].

The study design was chosen to compare acute complication frequencies in a few animals and without the need of a longitudinal study. A maximum of 30 biopsies, including sampling from the free ventricular wall, was performed to ensure adequate statistical power while reducing the required number of animals. As a result, only acute and major complications such as cardiac wall perforation and acute arrhythmias were evaluated. Although clinically relevant, long-term complications such as valve dysfunction or myocardial scarring were not assessed. Complications related to the vascular access and non-cardiac complications such as pneumothorax or stroke were also not considered. Since the aim of the study was to compare complication frequencies (in a highrisk scenario), not to estimate mortality risks, tamponade was considered an endpoint and no attempt was made to treat the animals (e.g., by pericardiocentesis). Moreover, the study was performed in an animal model and the sampling scheme was vastly different from standard clinical protocols. Consequently, the results cannot be used to estimate risks in a standard clinical scenario, where concurrent EMB has a significantly lower rate of major complications compared to the numbers presented in this study. Since the biopsy location was also varied, the complication risks from specific biopsy locations could not be evaluated.

It should also be noted that the diagnostic utility of microEMB depends on advances in molecular-based methods for diagnosis, since the samples are too small for conventional histology. In previous studies, we showed the capabilities to generate high quality RNA data from swine micro-biopsy samples, as well as detecting tissue changes after myocardial infarction [10, 11]. Moreover, Halloran and colleagues have developed and explored a gene expression profile test for allograft rejection, based on standard EMB samples [18].
The clinical utility of these gene expression tests, particularly from micro-biopsy samples, are yet to be investigated in clinical trials. Although these considerations limit the immediate translational outlook of the study, the safety comparison between micro-EMB and standard EMB is still relevant since micro-EMB is intended as a future alternative to EMB (within and beyond the current clinical indications).

Another important limitation of the study is the lack of blinding, which causes inevitable bias. Since the handling and appearance of the devices are fundamentally different, it was not possible to blind the operator. Nevertheless, all biopsies were performed by the same operator to avoid issues related to operator experience.

In conclusion, this study shows a safety advantage of microEMB compared to EMB in the employed animal model, consisting of high-risk sampling from several areas, including the free ventricular wall. This suggested safety advantage in highrisk scenarios has the potential to widen the diagnostic utility of myocardial biopsies in cases where molecular-based tests can replace or complement histology. Future studies should focus on long-term safety of biopsies from specific targets, including non-acute complications such as valve injuries. The sampling protocols should also be closer to real-world clinical scenarios, and preferably using a clinical grade micro-EMB device. Future studies should also assess the diagnostic capabilities of micro-EMB, as discussed elsewhere [11].

Supplementary Information The online version contains supplementary material available at https://doi.org/10.1007/s00380-021-01995-9.

Acknowledgements We acknowledge Karolinska Experimental Research and Imaging Centre (KERIC), Pellina Jansson, and Johanna Doshé, for providing facilities and technical assistance for the animal procedures. We acknowledge the Astrid Fagraeus laboratory for animal husbandry.

Author contributions All authors participated in the conception and design of the study and/or data acquisition and analysis, as well as writing and revising the manuscript.

Funding Open access funding provided by Karolinska Institute. The Erling Persson Family Foundation (Stockholm, Sweden), The Söderberg Foundations (Stockholm, Sweden), MedTechLabs (Stockholm, Sweden), and Karolinska Institutet (Stockholm, Sweden). The funders did not have any role in the design of the study or collection, analysis, and interpretation of data or in writing the manuscript.

\section{Declarations}

Conflict of interest The authors AC, MSa, RG, MSe, and SH are board members and/or shareholders of Microcardix $\mathrm{AB}$, which holds pending patents for the micro-EMB device used in this study. The other authors report no competing interests.

Human subjects/informed consent statement No human studies were carried out by the authors for this article. 
Animal studies The work had prior ethical approval from the local ethics committee (Stockholms Norra Djurförsöksetiska Nämnd, Stockholm, Sweden) and was conducted in accordance with local and national ethical guidelines, as well as the ARRIVE guidelines.

Open Access This article is licensed under a Creative Commons Attribution 4.0 International License, which permits use, sharing, adaptation, distribution and reproduction in any medium or format, as long as you give appropriate credit to the original author(s) and the source, provide a link to the Creative Commons licence, and indicate if changes were made. The images or other third party material in this article are included in the article's Creative Commons licence, unless indicated otherwise in a credit line to the material. If material is not included in the article's Creative Commons licence and your intended use is not permitted by statutory regulation or exceeds the permitted use, you will need to obtain permission directly from the copyright holder. To view a copy of this licence, visit http://creativecommons.org/licenses/by/4.0/.

\section{References}

1. Leone O, Veinot JP, Angelini A, Baandrup UT, Basso C, Berry G, Bruneval P, Burke M, Butany J, Calabrese F, d'Amati G, Edwards WD, Fallon JT, Fishbein MC, Gallagher PJ, Halushka MK, McManus B, Pucci A, Rodriguez ER, Saffitz JE, Sheppard MN, Steenbergen C, Stone JR, Tan C, Thiene G, van der Wal AC, Winters GL (2012) 2011 Consensus statement on endomyocardial biopsy from the Association for European Cardiovascular Pathology and the Society for Cardiovascular Pathology. Cardiovasc Pathol 21:245-274

2. Cooper LT, Baughman KL, Feldman AM, Frustaci A, Jessup M, Kuhl U, Levine GN, Narula J, Starling RC, Towbin J, Virmani R (2007) The role of endomyocardial biopsy in the management of cardiovascular disease. Circulation 116:2216-2233

3. Ammirati E, Frigerio M, Adler ED, Basso C, Birnie DH, Brambatti M, Friedrich MG, Klingel K, Lehtonen J, Moslehi JJ, Pedrotti P, Rimoldi OE, Schultheiss H-P, Tschöpe C, Cooper LT, Camici PG (2020) Management of acute myocarditis and chronic inflammatory cardiomyopathy: an expert consensus document. Circ Heart Fail 13(11):e007405

4. Singh V, Mendirichaga R, Savani GT, Rodriguez A, Blumer V, Elmariah S, Inglessis-Azuaje I, Palacios I (2017) Comparison of utilization trends, indications, and complications of endomyocardial biopsy in native versus donor hearts (from the Nationwide Inpatient Sample 2002 to 2014). Am J Cardiol 121(3):356-363

5. Khan T, Selvakumar D, Trivedi S, Rao K, Harapoz M, Thiagalingam A, Denniss AR, Varikatt W (2017) The value of endomyocardial biopsy in diagnosis and guiding therapy. Pathology 49(7):750-756

6. Chimenti C, Frustaci A (2013) Contribution and risks of left ventricular endomyocardial biopsy in patients with cardiomyopathiesclinical perspective: a retrospective study over a 28 -year period. Circulation 128:1531-1541

7. Bennett MK, Gilotra NA, Harrington C, Rao S, Dunn JM, Freitag TB, Halushka MK, Russell SD (2013) Evaluation of the role of endomyocardial biopsy in 851 patients with unexplained heart failure from 2000-2009. Circ Heart Fail 6:676-684
8. Cooper LT (2013) Role of left ventricular biopsy in the management of heart disease. Circulation 128:1492-1494

9. Giraldeau G (2018) The right way to the left ventricle: a better approach to endomyocardial biopsy? Can J Cardiol 34:1247-1249

10. Grankvist R, Chireh A, Sandell M, Mukarram AK, Jaff N, Berggren I, Persson H, Linde C, Arnberg F, Lundberg J, Ugander M, La Manno G, Jonsson S, Daub CO, Holmin S (2020) Myocardial micro-biopsy procedure for molecular characterization with increased precision and reduced trauma. Sci Rep 10:8029

11. Chireh A, Grankvist R, Sandell M, Mukarram AK, Arnberg F, Lundberg J, Daub CO, Holmin S (2021) Micro-biopsy for detection of gene expression changes in ischemic swine myocardium: a pilot study. PLoS ONE 16:e0250582

12. Yilmaz A, Kindermann I, Kindermann M, Mahfoud F, Ukena C, Athanasiadis A, Hill S, Mahrholdt H, Voehringer M, Schieber M, Klingel K, Kandolf R, Böhm M, Sechtem U (2010) Comparative evaluation of left and right ventricular endomyocardial biopsy: differences in complication rate and diagnostic performance. Circulation 122:900-909

13. Caforio ALP, Pankuweit S, Arbustini E, Basso C, Gimeno-Blanes J, Felix SB, Fu M, Heliö T, Heymans S, Jahns R, Klingel K, Linhart A, Maisch B, McKenna W, Mogensen J, Pinto YM, Ristic A, Schultheiss H-P, Seggewiss H, Tavazzi L, Thiene G, Yilmaz A, Charron P, Elliott PM (2013) Current state of knowledge on aetiology, diagnosis, management, and therapy of myocarditis: a position statement of the European Society of Cardiology Working Group on Myocardial and Pericardial Diseases. Eur Heart J 34:2636-2648

14. Seferović PM, Tsutsui H, McNamara DM, Ristić AD, Basso C, Bozkurt B, Cooper LT, Filippatos G, Ide T, Inomata T, Klingel K, Linhart A, Lyon AR, Mehra MR, Polovina M, Milinković I, Nakamura K, Anker SD, Veljić I, Ohtani T, Okumura T, Thum T, Tschöpe C, Rosano G, Coats AJS (2021) Starling RC Heart Failure Association of the ESC, Heart Failure Society of America and Japanese Heart Failure Society Position statement on endomyocardial biopsy. Eur J Heart Fail 3(6):854-871

15. From AM, Maleszewski JJ, Rihal CS (2011) Current status of endomyocardial biopsy. Mayo Clin Proc 86:1095-1102

16. Kiamanesh O, Toma M (2020) The state of the heart biopsy: a clinical review. CJC Open 3(4):524-531

17. Law YM, Lal AK, Chen S, Čiháková D, Cooper LT, Deshpande S, Godown J, Grosse-Wortmann L, Robinson JD, Towbin JA (2021) Diagnosis and management of myocarditis in children: a scientific statement from the American Heart Association. Circulation 144(6):e123-e135

18. Halloran PF, Potena L, Van Huyen J-PD, Bruneval P, Leone O, Kim DH, Jouven X, Reeve J, Loupy A (2017) Building a tissuebased molecular diagnostic system in heart transplant rejection: the heart Molecular Microscope Diagnostic (MMDx) System. J Heart Lung Transplant 36:1192-1200

Publisher's Note Springer Nature remains neutral with regard to jurisdictional claims in published maps and institutional affiliations. 